\title{
Dynamic Modeling COVID-19 for Comparing Containment Strategies in a Pandemic Scenario
}

\author{
Min Lu* \\ Department of Public Health, University of Miami, USA
}

*Corresponding author: Min Lu, Department of Public Health, University of Miami, USA.

Received Date: April 05, 2020

Published Date: May 18, 2020

\begin{abstract}
Since instances of coronavirus disease 2019 (COVID-19) community spread emerged in the United States, federal and local governments have implemented multiple containment measures. However, in order to satisfy the needs of citizens, the strictest containment measures can be only executed for short period. This article compares two types of containment strategies: a constant containment strategy that could satisfy the needs of citizens for a long period and an adaptive containment strategy whose strict level changes across time. When to implement the strictest measures is also of interest. A prediction model is proposed and a simple tool is developed for policy makers to compare different containment strategies. As an example, a county with 2.8 million populations with initial 200 infected cases is considered, where about $0.2 \%$ people dead during the pandemic. Compared with a constant containment strategy, adaptive containment strategies shorten the outbreak length, but executing the strictest measures late, even with stricter overall containment measures, will cause more mortality.
\end{abstract}

Keywords: Containment measures;; Incubation period; Infectious period; Pandemic; Period of communicability; Survival function.

\section{Introduction}

To prevent the spread of a new respiratory disease - coronavirus disease 2019 (COVID-19), policy makers rely on prediction models to foresee the dynamic of infected cases and prepare for adopting containment measures including patient quarantine, active monitoring of contacts, border controls, and community education and precautions [1-4]. There are many prediction models available for the COVID-19 pandemic [5-7,6-11,12,13,14]. To apply them for predicting local COVID-19 spread, there are two major challenges. Firstly, number of actual infected cases is usually unconfirmed and could be far larger than confirmed cases because there is significant number of infected cases in incubation period and test kits may be insufficient. On the other hand, regions that experienced earlier outbreaks can provide valuable information, such as the distribution of cure time, death time, and mortality rate [15], but it is not easy to integrate these dynamic parameters into most of the current models.

This article provides a simple and robust model framework whose parameters are dynamically adjustable and generally inter pretable for policy makers. The model allows unconfirmed infected cases and confirmed infected cases have different transmissibility. Survival analysis is integrated in it to borrow information from regions that experienced earlier outbreaks. Moreover, the model enables containment measures to change over time [16] through introducing a novel reproduction number which incorporate containment measures and the basic reproduction number $\left(\mathrm{R}_{0}\right)$.

\section{The Model}

Assume the disease of interest has an M-day period of communicability so that infected people are either cured or dead within $\mathrm{M}$ days. Denote the mortality rate within an infectious period as $m_{\text {death }}$ and the cure rate will be $1-m_{\text {death }}$. On day $\mathrm{t}$, denote the number of people that have been infected for d days as $p_{t, d}$ and the total number of infected cases is $\mathrm{Pt}=\mathrm{PM} \mathrm{d}=1 p_{t, d} \cdot p_{t, d}$ is determined by the following factors: 
- Mortality rate for people that have been infected for d days, denoted as $m_{d}$,

- Cure rate for people that have been infected for d days, denoted as $c_{d}$,

- number of people that an infected person can communicate on day t: when an unconfirmed infected case (for the reason of incubation period or insufficient test kits) pass the disease, it is denoted as reproductive number $R_{t}^{\text {unconfirmed }}$; for a confirmed infected case, it is denoted as $R_{t}^{\text {confirmed }}$ and $R_{t}^{\text {confirmed }}<R_{t}^{\text {unconfirmed }}$ because the person will be either hospitalized or quarantined at home with extra care,

- Test rate on day $\mathrm{t}$, denoted as $r_{t}^{\text {test }}$, which means that among the newly infected cases on day $\mathrm{t},\left(100 \%-r_{t}^{\text {test }}\right)$ of them are unconfirmed infected cases and $r_{t}^{\text {test }}$ of them are confirmed infected cases,

- And the number of travelers from other areas who have been infected for d days, denoted as $p_{t, d}^{i m p}$.

When moving forward from day t to $t+1$, number of people who have been infected for d days (on the $\mathrm{d}_{t h}$ day in their periods of communicability), $p_{t+1, d}$, is the sum of the number of survived but uncured cases from day $t$, the number of newly infected cases and the number of imported cases, denoted as $P_{t}=\sum_{d=1}^{M} P_{t, d}$ $[17,18,19]$ :

$$
P_{t+1}=\sum_{d=1}^{M} \rho_{t+1, d}=\sum_{d=1}^{M-1} \rho_{t, d}\left(1-m_{d}-c_{d}\right)+P_{t} r_{t}^{\text {test }} R_{t}^{\text {confirmed }}+P_{t}\left(1-r_{t}^{\text {test }}\right) R_{t}^{\text {unconfirmed }}+P_{t+1}^{\text {imp }}
$$

Note that people who have been infected for M days $\left(p_{t, M}\right)$ won't affect $P_{t+1}$ since their period of communicability will be over and they will be either dead or cured.

\section{Parameter Specification}

To specify mortality rate, a cumulative distribution function $F_{\text {death }}(t)=\mathrm{P}\left(T_{d} \leq t\right)$ is defined in interval $[0, M]$ for death time $T_{d}$ and $F_{\text {death }}(M)=m_{\text {death }}$. A lognormal distribution function is used as $F_{\text {death }}(t)=\frac{1}{2}+\frac{1}{2} \operatorname{erf}\left\lceil\frac{\ln t-\text {, where }}{\Gamma_{-}}\right.$ $\operatorname{erf}(x)=\frac{2}{\sqrt{\pi}} \int_{0}^{x} e^{-t^{2}} d t$.

. Here, parameters are set as $\sigma=0.8$ and $\mu=\ln (M)-\sqrt{2} \sigma \operatorname{erf}^{-1}\left(2 m_{\text {death }}-1\right)$ where $\operatorname{erf}^{-1}(x)$ denotes the inverse function of $\operatorname{erf}(x$; A patient has the probability of dying from day to $d+1$ as

$$
m_{d}=\mathrm{P}(d<T<d+1)=F_{\text {death }}(d+1)-F_{\text {death }}(d)
$$

Similarly, cure rate is modeled as $c_{d}=F_{\text {cure }}(d+1)-F_{\text {cure }}(d)$ , where $F_{\text {cure }}(t)=\mathrm{P}\left(T_{c} \leq t\right)$ is defined in interval $[0, M]$ for cure time $T_{c}$ and $F_{\text {cure }}(M)=1-m_{\text {death }}$. After specifying $F_{\text {cure }}(t)=\frac{1}{2}+\frac{1}{2} \operatorname{erf}\left[\frac{\ln t-\mu_{c}}{\sqrt{2} \sigma_{c}}\right] \quad$ we $\quad$ set $\quad \sigma_{c}=0.4 \quad$ and $\mu_{c}=\ln (M)-\sqrt{2} \sigma_{c} e r f^{-1}\left(1-2 m_{\text {death }}\right)$. For initial time, set $F_{\text {death }}(0)=F_{\text {cure }}(0)=0$.

The reproductive numbers $R_{t}^{\text {unconfirmed }}$ and $R_{t}^{\text {confirmed }}$ are determined by the basic reproduction number $R_{0}$, the containment measures on day and the percentage of uninfected people. It is assumed that cured cases will not get infected again since they are immune to the disease. Since $R_{0}$ is a constant, we only need to set

$$
R_{t}^{\text {unconfirmed }}=r_{t} \times \frac{P_{p o p}-P_{t}-\sum_{i=1}^{t}\left(D_{i}+C_{i}\right)}{P_{p o p}}
$$

where $D_{i}=\sum_{d=2}^{M} \rho_{i-1, d} m$ is the number of deaths on day $t=i, C_{i}=\sum_{d=2}^{M} p_{i-1, d} C$ is the number of cured patients on day $t=i$, and $P_{p o p}$ denotes the total population. The crucial parameter is which is used to specify the containment scenario. Set $R_{t}^{\text {confirmed }}=k \times R_{t}^{\text {unconfirmed }}$, where $k \in(0,1)$.

For initialization, infected durations are generated from Poisson distribution to mimic the individual variation [20], where $p_{1, d}=\sum_{i=1}^{P_{1}} 1_{X_{i}}=d$ and $p_{t, d}^{i m p}=\sum_{j=1}^{p_{t}^{i m p}} 1_{X_{j}=d} \quad X_{i} s$ and $X_{j} s$ are identically and independently distributed from a Poisson distribution with mean $\lambda$. When the generated value is zero or larger than $M$ , it is set as 1 or $M$.

\section{Results and Conclusion}

To compare different containment strategies, suppose a county is going to experience a COVID 19 outbreak in the scenario illustrated in Table 1. After monitoring 100 simulation replications, the dynamic of infected cases does not change much from random initialization. (Figure 1) In total, numbers of deaths from strategies $\mathrm{A}$, $B$ and $C$ are $5.34 \times 10^{3}, 4.99 \times 10^{3}$ and $5.61 \times 10^{3}$; numbers of infected cases are $1.87 \times 10^{5}, 1.75 \times 10^{5}$ and $1.97 \times 10^{5}$. The number of infected cases, $\mathrm{Pt}$, reaches its peak on the 42 th, 36 th and 35 th day and the

number of deaths, $D_{t}$, reaches its peak on the $66^{\text {th }}, 59^{\text {th }}$ and $60^{\text {th }}$ day from strategies A, B and C. After the peak of $P_{t}$, containment strategy does not make much difference on the trend of $P_{t}$ or $D_{t}$.

As a conclusion, compared with a constant containment strategy, adaptive containment strategies shorten the outbreak length. In order to achieve lower death rate, the strictest measures should be implemented two weeks before the peak of infected cases, instead of executing them during the peak. Adaptive strategy is less strict at 
the beginning, which results more severe spread. Even that, the following stricter measures effectively shortens the outbreak length [20]. When to choose the strictest measures is critical to achieve minimum total death rate, which is highly affected by the peak of predicted daily infected case under a constant containment strategy. Implementing the strictest measures late even with stricter overall containment measures, will cause more mortality.

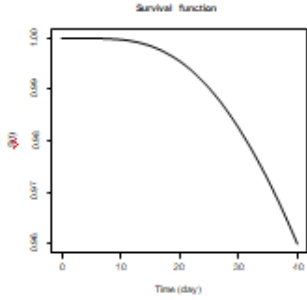

(a)

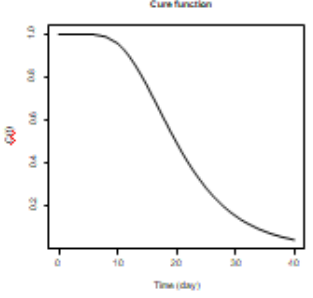

(b)

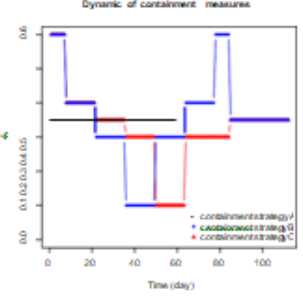

(c)

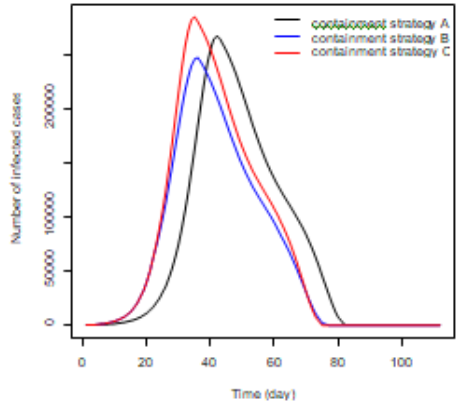

(d)

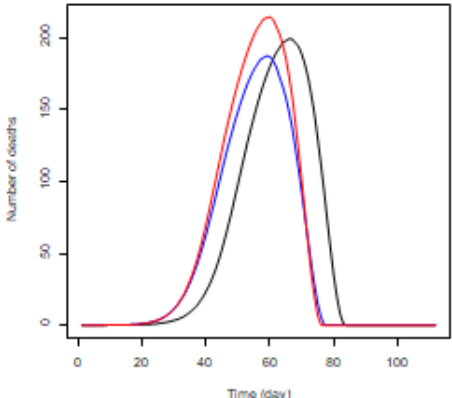

(e)

Figure 1: Containment strategy comparison from inputs illustrated in Table 1. Survival and cure functions with $4 \%$ mortality rate within 40 days are plotted in sub-figures (a) and (b). Sub-figure (c) demonstrates the strict level of containment strategies across time. Strategy A (black) has constant strict level while level of strictness can change weekly from strategies B (blue) and $C$ (red). Strategy $C$ implements the strictest measures two weeks earlier than strategy $B$. The averages of $r$ for strategies $A$ and $B$ are both 0.35 , and the average of $r$ for strategy $C$ is 0.325 , which means that strategy $C$ is overall stricter. From sub-figure (d) and (e), we can see that strategy $C$ results in the largest number of infected patients and deaths, followed by A and B. More adaptive containment strategies, B and C, ends the outbreak faster.

Table 1: Necessary inputs for policy makers to compare different containment strategies.

\begin{tabular}{|c|c|c|}
\hline Domain & Value & Description \\
\hline \multirow{5}{*}{ Disease } & $M=40$ & Infected cases will be either cured or dead within $M$ days. \\
\hline & $m_{\text {death }}=4 \%$ & Within $M_{\text {days, }} m_{\text {death }}$ of infected cases will be dead. \\
\hline & $r_{t}^{\text {test }}=(30+0.3 t) \%$ & On day $t, r_{t}^{\text {test }}$ of newly infected cases are tested for virus. \\
\hline & $\sigma_{c}=0.8$ & Parameter to shape the distribution function of death time. \\
\hline & $\sigma_{c}=0.8$ & Parameter to shape the distribution function of cure time. \\
\hline \multirow{4}{*}{ People } & $P_{p o p}=2.8 \times 10^{6}$ & On day $1, P_{p o p}$ individuals are not infected within the region. \\
\hline & $P_{1}=200$ & On day $1, P_{1}$ individuals are infectious. \\
\hline & $\begin{array}{l}P_{15}^{i m p}=P_{48}^{i m p}=2 \\
P_{29}^{i m p}=P_{63}^{i m p}=4\end{array}$ & $\begin{array}{c}\text { On day } 15,29,48 \text { and } 63 \text {, there are two, four, two and four infectious people who } \\
\text { travel into the region. }\end{array}$ \\
\hline & $\lambda=10$ & $\begin{array}{c}\text { Initial infectious cases, counted in } P_{1} \text { and } P_{t}^{i m p} \text {, have been infected for } \lambda \text { days on } \\
\text { average. }\end{array}$ \\
\hline
\end{tabular}




\begin{tabular}{|c|c|c|}
\hline \multirow{3}{*}{ Policy } & $r_{t}$ described in Figure 1(c) & Smaller value represents stricter containment measures*. \\
\cline { 2 - 3 } & $k=0.1$ & $k=R \leq_{t}^{\text {unconfirmed }} / R_{t}^{\text {confirmed }}$ \\
\hline
\end{tabular}

* $r_{t}$ can be interpreted as the average number of newly infected case communicated per infectious person per day on day , if nearly all the population are uninfected. For example $r_{t}=0.35$ from strategy A, implies every 100 infectious cases will communicate to 35 individuals per day on average. The model will adjust these inputs with percentage of infected cases across time, which produces $R_{t}^{\text {unconfirmed }}$ and $R_{t}^{\text {confirmed }}$.

\section{Acknowledgement}

This work was supported by the National Institutes of Health [grant numbers R01 CA200987 and R01 HL141892].

\section{Conflict of Interest}

No conflict of interest.

\section{Supplement}

An online prediction toolis provided athttps://minlu.shinyapps. io/killCOVID19/, where $R_{t}=r_{t}^{\text {test }} R_{t}^{\text {confirmed }}+\left(1-r_{t}^{\text {test }}\right) R_{t}^{\text {unconfirmed }}$ and $R$ across time is available for every state and county in the United ${ }^{t}$ States at https://minlu.shinyapps.io/killCOVID19map/.

\section{References}

1. D J Hunter (2020) Covid-19 and the stiff upper lip-the pandemic response in the united kingdom. New England Journal of Medicine.

2. K Kupferschmidt, J. Cohen (2020) Will novel virus go pandemic or be contained? 367: 610-611.

3. Y Ng, Z Li, YX Chua, WL Chaw, Z Zhao, et al. (2020) Evaluation of the effectiveness of surveillance and containment measures for the first 100 patients with covid-19 in singapore-january 2-february 29 69(11): 307-311.

4. FM Shearer, R Moss, JMcVernon, J V.Ross, and JM McCaw (2020) Infectious disease pandemic planning and response: Incorporating decision analysis. PLoS Medicine 17(1).

5. CT Bauch, JO Lloyd-Smith, MP Coffee, AP Galvani (2005) Dynamically modeling sars and other newly emerging respiratory illnesses: past, present, and future. Epidemiology 16(6): 791-801.

6. V Capasso (2008) Mathematical structures of epidemic systems. Springer Science \&Business Media 97.

7. V Capasso, G. Serio (1978) A generalization of the kermack-mckendrick deterministic epi- demic model. Mathematical Biosciences 42(1-2): 43-61.

8. V Colizza, A Barrat, M Barthelemy, AJ Valleron, A Vespignani (2007) Modeling the worldwide spread of pandemicinfluenza: baseline case and containment interventions. PLoS medicine 4(1).
9. C Dye ,N Gay (2003) Modeling the sars epidemic.Science 300(5627): 1884-1885.

10. A Gray, D Greenhalgh, L Hu, X Mao, J Pan (2011) A stochastic differential equation sis epidemic model. SIAM Journal on Applied Mathematics 71(3): 876-902.

11. C Y Huang, CT. Sun, JL. Hsieh, H Lin (2004) Simulating sars: Small-world epidemio- logical modeling and public health policy assessments. Journal of Artificial Societies and Social Simulation 7(4).

12. Wm Liu, SA Levin, Y Iwasa (1986) Influence of nonlinear incidence rates upon the be- havior of sirs epidemiological models. Journal of mathematical biology 23(2): 187-204.

13. H Rahmandad, JSterman (2008) Heterogeneity and network structure in the dynamics of diffusion: Comparing agent-based and differential equation models. Management Science 54(5): 998-1014.

14. J Zhang, J Lou, Z Ma, J Wu (2005) A compartmental model for the analysis of sars trans- mission patterns and outbreak control measures in china. Applied Mathematics and Compu- tation 162(2): 909-924.

15. DL Wilson (1994) The analysis of survival (mortality) data: fitting gompertz, weibull, and logistic functions. Mechanisms of ageing and development 74(1-2): 15-33.

16.J Cohen, K Kupferschmidt (2020) Strategies shift as coronavirus pandemic looms 367: 962-963.

17. M Chinazzi, JT Davis, M Ajelli, C Gioannini, M Litvinova, S Merler, et al. (2020) The effect of travel restrictions on the spread of the 2019 novel coronavirus (covid-19) outbreak. Science.

18. SP Layne, JM Hyman, DM Morens, JK Taubenberger (2020) New coronavirus outbreak: Framing questions for pandemic prevention.

19. G Pacheco, J Bustamante-Castañeda, JG Caputo, M Jiménez Corona, SPonce De León (2020).Dispersion of a new coronavirus sars-cov-2 by airlines in 2020: Temporal estimates of the outbreak in mexico.

20. JO Lloyd Smith, SJ Schreiber, PE Kopp, WM Getz (2005) Super spreading and the effect of individual variation on disease emergence. Nature, 438(7066): 355-359. 